\title{
WORKING CONDITION OF CLAMPING CIRCUIT WHEN IGCT SWITCHES OFF AND OPTIMAL DESIGN OF ITS CIRCUIT PARAMETERS
}

\author{
Yang Song ${ }^{1,2}$, Cailin Wang ${ }^{1}$ \\ ${ }^{1}$ School of Automation and Information Engineering, Xi'an University of Technology, Xi'an, 710048, China \\ ${ }^{2}$ School of Electronic Engineering, Xi'an Shiyou University, Xi'an, 710065, China \\ Email: yangsong82 ys@yeah.net
}

\begin{abstract}
With the rapid development of electric power semi-conductor in recent years, highpower converter devices have been extensively applied in multiple industrial fields. In this study, the working condition of clamping circuit in the process of Integrated Gate Commutated Thyristor (IGCT) turn-off was analyzed. Through studying the design principles of clamping circuit, a design scheme for optimizing the parameters of clamping circuit was put forward to ensure the safe operation of circuit. Simulation experiments were carried out. The optimal design of parameters and the simulation results demonstrated that the scheme could effectively optimize the parameters. The comparison between the proposed parameter optimal method and the traditional one suggested that the former was superior.
\end{abstract}

Keywords: IGCT turn-off, Clamping Circuit; Parameter Optimization.

\section{Introduction}

Integrated Gate-Commutated Thyristor (IGCT) is a novel power electronic device featured by high voltage, large current and low conduction loss, which has been extensively applied in high-power and high-voltage fields $[1,2]$. It is the integration of GCT chip and gate driver by means of low inductance; therefore it has stable turn-off performance and low conduction loss. Featured by large current, high voltage, low conduction loss and simple control, it has a significant superiority in application. Studies on IGCT have been gradually deeper for its excellent performance. Many scholars have investigated the structure, working principle and characteristic parameters of IGCT [3-6]. In the actual situation, IGCT will produce large turn-off overvoltage when being turned off due to the serial buffer inductance and stray inductance in circuit. The existence of such turn-off overvoltage will cause serious electromagnetic interference and aggravate device loss, and even damage IGCT device.

The protection of IGCT also has been frequently studied. Bai J et al. [7] put forward a new buffer circuit which was used for three-phase current source inverter and a small auxiliary pulse buffer control strategy to effectively protect IGCT. Zou Y et al. [8] put forward a design of snubber circuit parameter to reduce device loss. Zhao Y et al. [9] designed the structure of hybrid circuit breaker to extend the service time of devices. Wang $C$ et al. [10] designed a protective system for IGCT and verified its superiority and reliability by experiments. In IGCT, clamp circuit is needed to suppress turn-off overvoltage. Design of parameter optimization of clamp circuit has been one of the key problems in IGCT studies. Many scholars have analyzed devices and parameters affecting IGCT turn-off, but there is no good design of clamp circuit currently. Based on the analysis on the working principles of clamp circuit, Wang J et al. [11] put forward a design method for precise improvement of clamp circuit parameters by optimizing nonlinear inequality constraint problems with fmincon function and verified its superiority through experiments. Tong Y [12] studied the drive technology involved in IGCT and designed a drive circuit for $4000 \mathrm{~A} / 4500 \mathrm{~V}$ series IGCT. In this study, a parameter optimization method was put forward based on the study of the working principles of IGCT, the analysis on the working state of clamp circuit in the process of IGCT turn-off and the calculation of the working states in different processes. Moreover simulation experiment was performed to verify the superiority of the method.

1. IGCT

IGCT is a novel power electronic device with excellent performance, which has replaced gate turnoff thyristor [13]. It is more efficient in electron extraction and turn-off and more stable. It integrates the advantages of GCT chip and gate driver; therefore it is featured by large current, high voltage, low conduction loss, compact structure and low cost. It has extensive application and a good application prospect. 
But it also has deficiencies. The allowed current variation rate is low in IGCT; hence the voltage spike may appear due to the stray inductance in some circuits when IGCT is turned off. Such a condition may induce shock, serious electromagnetic interference, increased device loss and even damages to devices. In view of this problem, additional clamp circuit is needed to suppress turnoff overvoltage.

\section{Analysis on the Working Condition of Clamp Circuit in the Process of IGCT Turn-Off}

Clamp circuit in IGCT is composed of buffer inductance (Li), clamp diode (DCL), clamp capacitor CCL and clamp resistance RCL. As shown in Figure 1, CDC stands for direct current capacitor, LLoad stands for load, D1 and D2 stand for IGCT and freewheel diode respectively, and LCL stands for stray inductance.

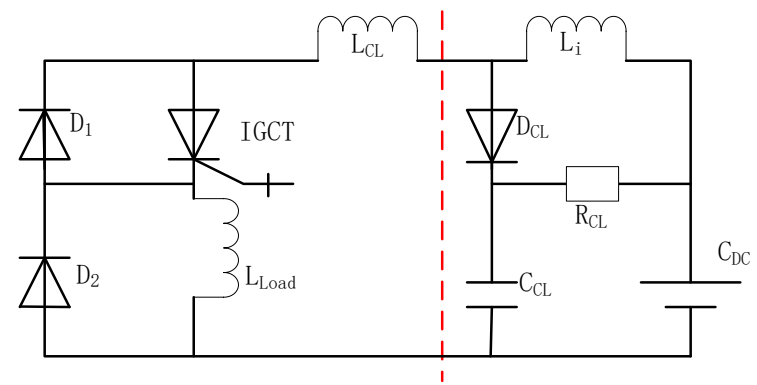

Figure 1: The basic circuit unit of IGCT

When IGCT is in an off state, the load current follows via D2; when it turns on, the current converts to IGCT. To prevent the damages caused by the rapid changes of current, buffer inductance $\mathrm{Li}$ is connected in series. Because of the existence of $\mathrm{Li}$ and LCL, IGCT will produce large voltage when being turned off; therefore clamp capacitor CCL and clamp resistance RCL are needed to suppress voltage.

Moreover to prevent short circuit, clamp diode DCL is also needed.

The operation state of the basic circuit in the process of IGCT turn-off is shown in Figure 2. $\mathrm{uL}(\mathrm{t})$ and $i L(t)$ stand for the instantaneous voltage at both ends of buffer induction $\mathrm{Li}$ and the instantaneous current flowing through Li respectively, $\mathrm{uC}(\mathrm{t})$ and $\mathrm{iC}(\mathrm{t})$ stand for the instantaneous voltage and current at both ends of clamp capacitor CCL respectively, UD and UT stand for the voltage of direct-current power supply CDC and IGCT respectively, and $i R(t)$ and IL stand for the instantaneous current passing through clamp resistance RCL and load LLoad respectively.

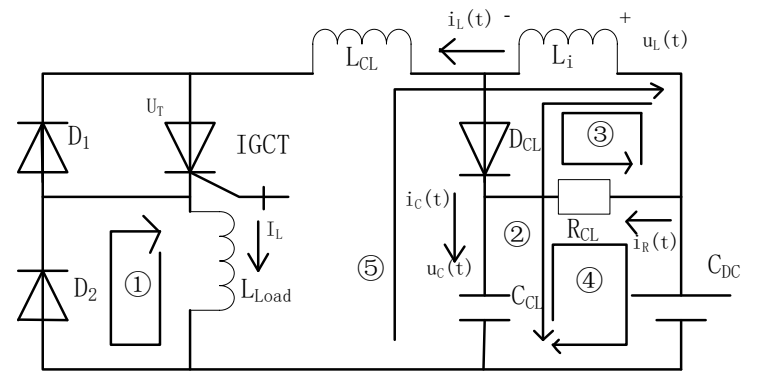

Figure 2: The operation state of circuit when IGCT turns off

In the process of IGCT turn-off, the operation of clamp circuit can be divided into three stages. T1, T2 and T3 stand for the action time of the three stages respectively.

In the first stage, the voltage increases, the current passing through LCL immediately converts to DCL, and Li refluxes via (2) and (3).

In the second stage, inductance Li and capacitor CCL discharge. At that moment, inductive current $\mathrm{uC}(\mathrm{t})$ and capacitor voltage $\mathrm{iL}(\mathrm{t})$ both decreased to 0 . In the period, iL(t) consumes energy via RCL, forming loop (3); CCL discharges via RCL, forming loop (4).

In the third stage, capacitor CCL discharges, DCL recovers reversely. DCL discharges via loop(5); The voltage at both ends increases firstly and then decreased.

In the process of operation, repetitive peak offstate voltage UDRM of IGCT and repetitive peak reverse voltage URRM of the diode which is antiparalleled with IGCT exist. In the process of turn-off, the maximum terminal voltage of IGCT cannot exceed the smaller one among UDRM and URRM; otherwise, IGCT may be damaged. Moreover clamp capacitor will absorb the energy of buffer inductance after IGCT turns off and must be thoroughly released before the next turn-off. The period from clamp circuit starting to work after IGCT turn-off to clamp capacitor recovering to the initial value before turnoff is the transient process time of clamp circuit Ts.

The smaller the value of Ts is, the higher the theoretical switching frequency of IGCT is.

In the first and second stages, the terminal voltage of IGCT was equal to the instantaneous value of the terminal voltage of clamp capacitor CCL. Therefore the value of UDM can be obtained through analyzing the instantaneous value of the terminal voltage of CCL. According to the basic circuit principles, the formula of the terminal voltage of clamp diode $\mathrm{uC}(\mathrm{t})$ was:

$$
\frac{d^{2} u_{c}(t)}{d t^{2}}+\frac{1}{R_{C L} C_{C L}} \frac{d u_{c}(t)}{d t}+\frac{1}{L_{i} C_{C L}} u_{c}(t)=\frac{U_{D}}{L_{i} C_{C L}}
$$


$\boldsymbol{\delta}$ was defined as attenuation coefficient, $\boldsymbol{\omega}_{0}$ as natural frequency, and D as damping coefficient, then

$$
\left\{\begin{array}{c}
\delta=1 /\left(2 R_{C L} C_{C L}\right) \\
\omega_{0}=1 / \sqrt{L_{i} C_{C L}} \\
D=\delta / \omega_{0}=\sqrt{L_{i} / C_{C L}} /\left(2 R_{C L}\right)
\end{array}\right.
$$

The value of clamp capacitor voltage can be obtained according to the instantaneous voltage of $\mathrm{uc}_{\mathrm{C}}(\mathrm{t})$ during IGCT turn-off.

$$
u_{c}(t)=\left\{\begin{array}{c}
U_{D}+\frac{I_{L} e^{-D \omega_{0} t} \sin \left(\omega_{0} \sqrt{1-D^{2}}\right)}{C_{C L} \omega_{0} \sqrt{1-D^{2}}}, \\
D<1 \\
U_{D}+I_{L} e^{-\omega_{0} t} \cdot t / C_{C L}, D=1 \\
U_{D}+\frac{I_{L} e^{\omega_{0} t} \cdot\left[e^{\left(\omega_{0} \sqrt{D^{2}-1}\right) t}-e^{\left(-\omega_{0} \sqrt{D^{2}-1}\right) t}\right]}{2 C_{C L} \omega_{0} \sqrt{D^{2}-1}} \\
D>1
\end{array},\right.
$$

When the value of $\mathrm{D}$ varies, the solutions to the instantaneous voltage of clamp capacitor can be divided into direct component and alternating component. When the value of $\mathrm{D}$ is larger than 1 , alternating component will attenuate to 0 under the influence of sin function and exponential function, and next switching period will start. Therefore it is reasonable to design clamp circuit under the condition of $\mathrm{D}<1$.

The maximum value of the terminal voltage of clamp capacitor can be obtained by taking the derivative of equation (3) under the condition of D < 1.

$U_{C \max }=U_{D}+\frac{I_{L}}{C_{C L} \omega_{0}} e^{-\left(D / \sqrt{1-D^{2}}\right) \arctan \left(\sqrt{1-D^{2}} / D\right)}$

When $\mathrm{D}<1$, the action time in the first stage of the turn-off process is:

$$
T_{1}=\arctan \left(\sqrt{1-D^{2}} / D\right) /\left(\omega_{0} \sqrt{1-D^{2}}\right)
$$

Then the equation of the instantaneous value of inductive current $i_{L}(t)$ is:

$$
\frac{d^{2} i_{L}(t)}{d t^{2}}+\frac{1}{R_{C L} C_{C L}} \frac{d i_{L}(t)}{d t}+\frac{i_{L}(t)}{L_{i} C_{C L}}=0
$$

The initial value of $i_{L}(t)$ is load current $I_{L}$. When $\mathrm{D}<1$, we have:

$$
i_{L}(t)=\frac{I_{L} e^{-D \omega_{0} t} \cdot \sin \left[\omega_{0} \sqrt{1-D^{2}} \cdot t+\arctan \left(\frac{\sqrt{1-D^{2}}}{D}\right)\right]}{\sqrt{1-D^{2}}}
$$

The action time in the second stage is:

$$
T_{2}=\frac{\pi-2 \arctan \left(\frac{\sqrt{1-D^{2}}}{D}\right)}{\omega_{0} \sqrt{1-D^{2}}}
$$

The transient time of IGCT turn-off process is:

$$
T_{s}=T_{1}+T_{2}=\left[\pi-\arctan \left(\sqrt{1-D^{2}} / D\right)\right] /\left(\omega_{0} \sqrt{1-D^{2}}\right)
$$

\section{Parameter Design of Clamp Circuit}

Let the relative overvoltage on $\mathrm{C}_{\mathrm{CL}}$ be $\Delta u_{c}(t)$. The maximum value of $\Delta u_{c}(t)$ in the process of turn-off is $\Delta u_{C \max }$, which should be smaller than the allowable relative overvoltage of the device $\Delta U_{C L \max }$. Then

$$
\Delta u_{C \max }=\frac{I_{L}}{C_{C L} \omega_{0}} e^{-\left(D / \sqrt{1-D^{2}}\right) \arctan \left(\sqrt{1-D^{2}} / D\right)} \leq \Delta U_{C L \max }
$$

Substitute equation (2) into equation (10), then

$$
C_{C L} \geq \frac{L_{i} I_{L}^{2}}{\Delta U_{C L \max }^{2}} e^{-\left(2 D / \sqrt{1-D^{2}}\right) \arctan \left(\sqrt{1-D^{2} / D}\right)}
$$

When the buffer inductive current decreased to 0 , the relative overvoltage on clamp capacitor decreased to $5 \%$ of the peak value, then

$$
\frac{\Delta u_{C}(t)_{t=T_{S}}}{\Delta u_{C \max }}=\frac{e^{-D \omega_{0}\left(T_{1}+T_{2}\right)} \sin \left\lfloor\omega_{0} \sqrt{1-D^{2}} \cdot\left(T_{1}+T_{2}\right)\right\rfloor}{\sqrt{1-D^{2}} \cdot e^{-\left(D / \sqrt{1-D^{2}}\right) \arctan \left(\sqrt{1-D^{2}} / D\right)}}=0.05
$$

$$
D \approx 0.835
$$

Substitute equation (13) into equation (11), and then the design formula of clamp capacitor can be obtained.

$$
C_{C L} \geq 0.171 L_{i} I_{L}^{2} / \Delta U_{C L \max }^{2}
$$

The design formula of clamp resistance can be obtained according to the definition and assignment of D.

$$
R_{C L}=\frac{\sqrt{L_{i} / C_{C L}}}{2 D}=0.599 \sqrt{L_{i} / C_{C L}}
$$




\section{Simulation and Experimental Verification}

The repetitive peak off-state voltage of IGCT $U_{D R M}$ was $4500 \mathrm{~V}$, the maximum current ${ }{ }_{r}$ was $630 \mathrm{~A}$, the maximum switching current variation rate $d i / d t_{I G C T}$ was $300 \mathrm{~A} / \mu \mathrm{s}$, direct current capacitor was $3400 \mu \mathrm{F}$, and $70 \mu \mathrm{H}$ inductance coil was selected as load. Let the maximum value of direct voltage $U_{D}$ be $3300 \mathrm{~V}$, then

$$
\begin{aligned}
& L_{i} \geq U_{D} /\left(d i / d t_{I G C T}\right)=3300 / 330=11 \mu H \\
& \Delta U_{C L \max }=(2 / 3)(4500-3300)=800
\end{aligned}
$$

According to equation (14), clamp capacitor is:

$$
C_{C L} \geq \frac{0.171 \times 11.5 \times 630^{2}}{800^{2}}=1.22 \mu F
$$

In the traditional way, the value of clamp capacitor is $4.32 \mu \mathrm{F}$. About $70 \%$ of capacitance can be saved through optimizing the design of clamp capacitor.

According to equation (15),

$$
R_{C L}=0.599 \sqrt{11.5 / 2}=1.44 \Omega
$$

In the traditional way, the value of clamp resistance is $1.5 \Omega$. The clamp resistance after optimization is smaller than $1.5 \Omega$.

Then simulation analysis is performed on clamp circuit using IGCT physical model. The simulation and comparison of the terminal voltage of clamp capacitor and inductive current is shown in Figure 3 and 4 .

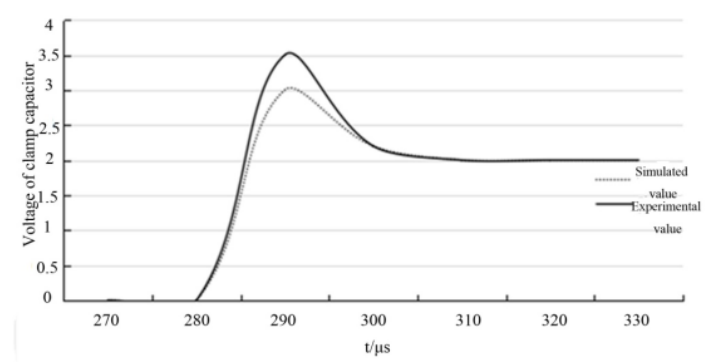

Figure 3: Comparison of terminal voltage of clamp capacitor

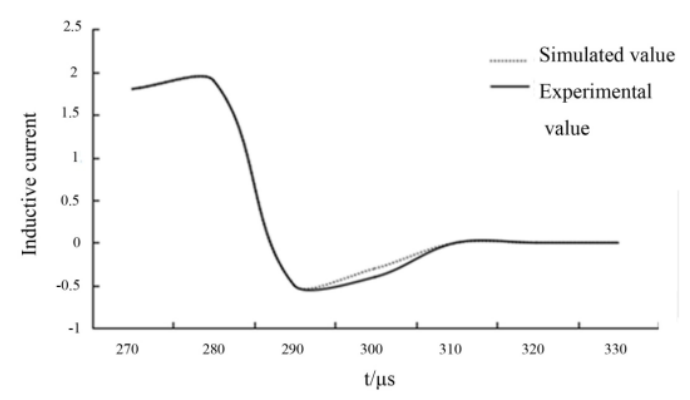

Figure 4: Comparison of inductive current
The comparison suggested that the gap between the simulated value and experimental value was quite small, indicating that the parameter optimization of clamp circuit had favorable accuracy and verifying the feasibility of the method. The difference between the simulated value and experimental value is mainly induced by the existence of stray inductance. Therefore optimal design of parameters should fully consider the influence of stray inductance. The disturbance of stray inductance should be minimized to keep a high accuracy of parameter design.

The calculation results suggested that clamp circuit designed through parameter optimization had $4.32 \mu \mathrm{F}$ of clamping capacitance and 1.5 of clamping resistance, which are lower than the clamping capacitance and resistance in the traditional design. The design saves the cost of hardware and improves working efficiency.

\section{Discussion and Conclusion}

IGCT is a novel power semiconductor switching device developed by ABB company in 1997. It integrates GTO chip, anti-parallel diode and gate driver together and connects by means of low inductance. It has the stable turn-off capability of diode and the low conduction loss of thyristor; hence it is a high-efficient and low-cost technology [14,15] and has an extensive application prospect in high power conversion [16]. Compared to other devices, IGCT has low voltage and strong current conductivity [17]. When IGCT turns off, the stray current of some parts of the circuit will generate voltage spike, leading to increased turn-off voltage, longer turn-off transient time and even damages to devices [18]. Relevant studies concerning the protection for safe operation of IGCT become increasingly deeper $[19,20]$. To solve the problem of power loss, Blinov A et al. [21] came up the idea of connecting insulated gate bipolar translator (IGBT) with IGCT to combine their advantages and reduce power loss. Addition of clamp circuit is a common way in the current studies.

The existence of clamp circuit can reduce device loss [22]. In the process of IGCT turn-off, voltage increases and convert to clamp diode, buffer inductance discharges, clamp capacitor charges, and clamp resistance consumes energy. After that, clamp capacitor begins to discharge, and clamp diode recovers reversely. In such a process, energy is consumed through clamp resistance, which reduces loss and moreover saves hardware cost.

The clamp capacitance is $1.22 \mu \mathrm{F}$ after optimization, which saved $70 \%$ compared to the traditional circuit. Moreover the clamp resistance of the optimized circuit was also smaller than that of the traditional circuit. Therefore the optimization method was feasible. 
IGCT has an extensive development prospect in the industrial field. Through the analysis on the working principles of clamp circuit in IGCT and the understanding on the working conditions in different stages, an optimal design of clamp circuit parameters was put forward. Moreover the validity and effectiveness of the method was verified by simulation and experiments, which can provide guidance for the parameter design and optimization of IGCT. The experiments also have deficiencies. The loss of circuit needs to be deeply studied and further optimized.

\section{References:}

[1] Rocha A V, França G J, Santos M E D, et al. Improving the performance of protection schemes in three level IGCT-based neutral point clamped converters. Energy Conversion Congress and Exposition. IEEE, 2010:2326-2332.

[2] Wang P, Li C, Ge G, et al. Research on rectifier system in high power IGCT three-level PWM converters. International Conference on Electrical Machines and Systems. IEEE, 2016:1541-1546.

[3] Kang T, Lim H, Suh Y. Loss analysis of current source converter for $10 \mathrm{kV}$ IGCT in 7MW PMSG wind turbine systems. IEEE, International Power Electronics and Motion Control Conference. IEEE, 2016:193-200.

[4] Li B, Xu X, Yin C. Research on three level high power four quadrant medium voltage inverter based on IGCT. IEEE Transportation Electrification Conference and Expo, Asia-Pacific. IEEE, 2017:1-6.

[5] Vemulapati U R, Bianda E, Torresin D, et al. A Method to Extract the Accurate Junction Temperature of an IGCT During Conduction Using Gate-Cathode Voltage. IEEE Transactions on Power Electronics, 2016, 31(8):5900-5905.

[6] Chen Z, Yu Z, Zhang X, et al. Analysis and Experiments for IGBT, IEGT, and IGCT in Hybrid DC Circuit Breaker[J]. IEEE Transactions on Industrial Electronics, 2018, 65(4):2883-2892.

[7] Bai J, Lu S, Liu J, et al. Research on snubber circuit for high-power IGCT Based On Cur Rent Source Inverter. International Conference on Mechatronic Sciences, Electric Engineering and Computer. IEEE, 2014:3152-3155.

[8] Zou Y, Zhenyu M A, Luo L, et al. Parameter Design of Snubber Circuit for Phase Model of IGCT Converter[J]. High Power Converter Technology, 2015, 5:18-22.

[9] Zhao Y, Feng Y. Research on hybrid circuit breaker based on IGCT \& current detection of neural network. International Forum on Strategic Technology. IEEE, 2011:1018-1022.

[10] Wang C, Li C, Zhu C, et al. Investigation on the protection system of the large power converter with IGCT. Power Electronics and Motion Control Conference. IEEE, 2012:1603-1606.

[11] Wang J, Kong L, Zhou Y, et al. Analysis and Parameters Design of Clamping Circuit for IGCT Converters. Zhongguo Dianji Gongcheng Xuebao/proceedings of the Chinese Society of Electrical Engineering, 2017, 37(15):4463-4471.

[12] Tong Y. Drive Technology for $4000 \mathrm{~A} / 4500 \mathrm{~V}$ Series IGCT. Transactions of China Electrotechnical Society, 2010, 25(8):110-115.

[13] Lan Z, Li C, Li Y, et al. The investigation on a novel IGCT-based cascade high voltage large capacity converter. International Conference on Electrical Machines and Systems. IEEE, 2011:1-4.

[14] Steimer P K, Gruning H E, Werninger J, et al. IGCT-a new emerging technology for high power, low cost inverters. Industry Applications Magazine IEEE, 1999, 5(4):12-18.

[15] Stiasny T, Kappatos V, Setz T, et al. Where Higher Power Handling Capability is RequiredIGCT is the Right Choice. High Power Converter Technology, 2015.6:1-7.

[16] Xu D, Han M X, Wan L. Power Loss Research on IGCT-applied NPC Three-level Converter[J]. Telkomnika Indonesian Journal of Electrical Engineering, 2014,12(7) : 5154-5162.

[17] Chen Z, Yu Z, Zhang X, et al. Analysis and Experiments for IGBT, IEGT, and IGCT in Hybrid DC Circuit Breaker. IEEE Transactions on Industrial Electronics, 2018, 65(4):2883-2892.

[18] Liu J, Zhenyu M A, Sun B. Optimal Design of Stray Inductance of Busbar in Two-level IGCT Power Module. High Power Converter Technology, 2015, 6:14-19.

[19] Lophitis N, Antoniou M, Udrea F, et al. The Destruction Mechanism in GCTs. IEEE Transactions on Electron Devices, 2013, 60(2):819-826.

[20] Rocha A V, Paula H D, Santos M E D, et al. A thermal management approach to fault-resilient design of three-level IGCT-based NPC converters. IEEE Energy Conversion Congress and Exposition. IEEE, 2013:1703-1710.

[21] Blinov A, Vinnikov D, Ivakhno V, et al. Hybrid IGBT-IGCT Switch. Przeglad Elektrotechniczny, 2012, 88(1):12-15.

[22] Alvarez R, Bernet S, Lindenmueller L, et al. Characterization of a new $4.5 \mathrm{kV}$ press pack SPT+ IGBT in Voltage Source Converters with clamp circuit. IEEE International Conference on Industrial Technology. IEEE, 2010:702-709. 